\title{
Electromagnetic Mass Shifts in Non-Lagrangian Field Theory
}

\author{
O. SteinmanN ${ }^{\star}$ \\ International Centre for Theoretical Physics, Miramare-Trieste
}

Received July 9, 1969

\begin{abstract}
The electromagnetic interactions of hadrons are considered in a model in which both the hadrons and the photons are spinless. The perturbation theory of this model is developed in a way which takes the strong interactions rigorously into account. An expression for the second-order electromagnetic mass shifts of the hadrons is derived. The customary formula expressing these mass shifts as integrals over the Compton scattering amplitude is shown to be inaccurate. The correct expression differs from the old one by a changed integration contour. In favourable cases the two expressions coincide if the integrations over the four components of the photon momentum are carried out in a certain order. This order of integration must in general not be changed.
\end{abstract}

\section{Introduction}

Attempts to calculate electromagnetic mass differences of hadrons to lowest order in the fine structure constant often start from the relation (here written for the meson case)

$$
\delta M^{2}=\frac{i}{2} \frac{e^{2}}{(2 \pi)^{4}} \int d^{4} q K_{\mu \nu}\left(q^{2}\right) M_{\mu \nu}(p, q),
$$

where $K_{\mu \nu}$ is the photon propagator and $M_{\mu \nu}$ is the second-order forward scattering amplitude of a photon with momentum $q$ (not necessarily on the mass shell) on a hadron of momentum $p$ (on the mass shell). This formula has been the source of some puzzlement in the last few years, since current algebra arguments seem to indicate that it leads to an infinite $\pi^{+}-\pi^{0}$ mass difference [1]. Even though these arguments are by no means compelling (see, e.g., Ref. 2 for pertinent criticism), it is evidently desirable to know to what extent Eq. (1.1) can be trusted.

Equation (1.1) would be an immediate consequence of the Feynman rules if the hadrons had no interactions apart from the electromagnetic ones. However, the strong interactions cannot be neglected in this problem and this makes a creditable derivation of a formula for $\delta M^{2}$ considerably

* Presently at: Schweizerisches Institut für Nuklearforschung, Zürich 
more difficult. Indeed, none of the existing derivations [3-7] can be considered to be fully satisfactory. Either they proceed formally, ignoring the problems connected with the (non-existent) definition of products of field operators in a point and assuming the interacting fields to satisfy canonical commutation relations, or they apply perturbation theory also to the strong interactions and show (1.1) to be correct to all orders in the strong coupling. In the work of Theis [7] the extent to which perturbation theory in the strong interaction is used has been reduced to a minimum, but even so the question of the probable divergence of the perturbation series remains.

In the present paper we propose to approach the problem within the LSZ framework of field theory, without recourse to Langrangians or canonical commutation relations. We shall develop a form of perturbation theory for a "weak" interaction of the electromagnetic type, which takes the strong interactions rigorously into account. Only mathematically well defined operations will be used. The formalism will be developed only up to second order.

Analogous formulations of customary perturbation theory (i.e., the variety starting from free fields as 0 th approximation) have been given by various authors. As relevant to our present purpose we mention the work by Nishijima [8] employing time-ordered products and two earlier papers by the present author [9] working with retarded functions.

Since some problems peculiar to electrodynamics (i.e., those connected with gauge invariance and the infra-red divergences) are not yet fully understood in axiomatic field theory, we shall not deal with electrodynamics in its actual form. Instead we shall consider a model with scalar photons which, moreover, have a small non-vanishing mass. This mass can be put equal to zero in the final result, provided that no infra-red divergences are thereby introduced. There is no doubt that our result will carry over to real electrodynamics.

We shall also simplify the hadronic part of the theory by assuming that only a single kind of hadrons exists, corresponding to a hermitian pseudoscalar field. This is merely a labour-saving device. The generalization to more realistic situations does not present any difficulties.

We shall find that the expression for $\delta M^{2}$ derived in this way is not (1.1) but differs from this by the shifting of an integration path. (1.1) is thus only correct if this shift is allowed.

The programme of the paper is as follows. In Section II the model under consideration will be stated and the field theoretic background will be explained. Perturbation theory to second order will be developed in Sections III-V. A formula for the electromagnetic mass shift will be derived in Section VI and discussed in Section VII; which latter section will also contain some miscellaneous remarks. 


\section{The Model}

We consider a theory of two local hermitian fields, the pseudoscalar "hadron" field $A(X)$ and the scalar "photon" field $B(x)^{1}$ satisfying the Wightman axioms $[10,11]$. If applied to the vacuum, the fields $A, B$ shall create one-particle states with masses $M$ and $m$ respectively, with $0<m \ll M$. We assume asymptotic completeness, i.e., the asymptotic fields $A^{\text {in }}, B^{\text {in }}$ shall generate the full Hilbert space $\mathfrak{H}$ of the theory. This means that $\mathfrak{H}$ is of the form

$$
\mathfrak{H}=\mathfrak{H}_{A} \otimes \mathfrak{H}_{B}
$$

where $\mathfrak{H}_{A}$ and $\mathfrak{H}_{B}$ are the Fock spaces of the free fields $A^{\text {in }}$ and $B^{\text {in }}$ respectively. The vacuum $\Omega$ of $\mathfrak{H}$ is the direct product of the partial vacua $\Omega_{A}$ and $\Omega_{B}$. The subspace $\mathfrak{G}_{A} \otimes \Omega_{B}$ will often be called $\mathfrak{H}_{A}$ by abuse of language. The hadrons shall be strongly interacting among themselves and shall interact with the photons via an interaction of electromagnetic type, i.e., an interaction which is linear in $B$ if expressed in Lagrangian terms. For a non-Lagrangian definition of this notion we refer to Section IV. The photons shall have no direct self-interaction. We assume the theory to depend on the $A-B$ coupling constant $e$ in such a way that all the requirements are still satisfied for $e=0$. In this limit $B$ is a free field of mass $m$ and the fields $A$ and $B$ are independent in the sense that application of any polynomial in $A$ to $\Omega$ yields a state in $\mathfrak{H}_{A}$. The parity assumptions on $A$ and $B$ ensure the automatic vanishing of the vacuum expectation value of $A(X)$ and of the mixed two-point function. The correct parity behaviour of the theory will not be discussed explicitly in what follows since it is trivial. The word "scalar" will from now on only denote the transformation character under the connected Lorentz group, i.e., it may stand for "scalar" or "pseudoscalar" as the case may be.

The LSZ formalism will be used as described in an earlier paper [12], hereafter referred to as $E$. Unless otherwise noted, the definitions and notations of $E$ will be used. Our main tools will be the retarded and timeordered functions as defined in $E$. Since we are now dealing with two fields there exist two sets $r\left(x_{1}, \ldots, x_{b}, X_{1}, \ldots, X_{a}\right)$ and $r\left(X_{1}, \ldots, X_{a}, x_{1}, \ldots, x_{b}\right)$ of retarded functions, depending on whether the distinguished first variable is a photon or a hadron variable. Amputation of $r$ with respect to the variable $X_{j}$ or $x_{h}$ will be denoted by a bar over this variable:

$$
\begin{aligned}
& r\left(\ldots, \bar{X}_{j}, \ldots\right)=-\left(\square_{X_{j}}+M^{2}\right) r\left(\ldots, X_{j}, \ldots\right), \\
& r\left(\ldots, \bar{x}_{h}, \ldots\right)=-\left(\square_{x_{h}}+m^{2}\right) r\left(\ldots, x_{h}, \ldots\right) .
\end{aligned}
$$

1 We shall always use capital letters for $A$-field variables, small letters for $B$-field variables. 
The GLZ equations [13] read in our case

$$
\begin{aligned}
& r\left(x, y, x_{1}, \ldots, x_{b}, X_{1}, \ldots, X_{a}\right)-r\left(y, x, x_{1}, \ldots, x_{b}, X_{1}, \ldots, X_{a}\right) \\
& =-i\left[\sum_{L} \sum_{\alpha, \beta=0}^{\infty} \frac{i^{\alpha+\beta}}{\alpha ! \beta !} \int \prod_{1}^{\alpha}\left\{d u_{h} d v_{h} \Delta_{+}\left(u_{h}-v_{h}\right)\right\} \prod_{1}^{\beta}\left\{d U_{j} d V_{j} \Delta_{+}\left(U_{j}-V_{j}\right)\right\}\right. \\
& \left.r\left(x, x_{L}, X_{L}, \bar{u}_{1}, \ldots, \bar{u}_{\alpha}, \bar{U}_{1}, \ldots, \bar{U}_{\beta}\right) r\left(y, x_{R}, X_{R}, \bar{v}_{1}, \ldots, \bar{v}_{\alpha}, \bar{V}_{1}, \ldots, \bar{V}_{\beta}\right)-(x \leftrightarrow y)\right]
\end{aligned}
$$

and similar equations obtained by replacing one or both of the variables $x, y$ by a hadron variable. The summation $\sum_{L}$ extends over all partitions of the set $\left(x_{1}, \ldots, X_{a}\right)$ into two complementary subsets $\left(x_{L}, X_{L}\right)$ and $\left(x_{R}, X_{R}\right)$, one of which may be empty. The singular functions $\Delta_{+}$have to be taken for the appropriate mass, i.e., $m$ for photons, $M$ for hadrons.

The backbone of our investigation is the GLZ theorem, which will be used in the following formulation:

Theorem 1. Let $r\left(x_{1}, \ldots, X_{a}\right), r\left(X_{1}, \ldots, x_{b}\right)$ be tempered distributions with the following properties:

a) Equations (2.3) are satisfied.

b) The $r$ are real, invariant under the connected Poincare group and symmetrical under permutations of all variables except the first one.

c) The support of $r\left(x, x_{1}, \ldots, x_{b}, X_{1}, \ldots, X_{a}\right)$ is contained in the set

$$
x-x_{h} \in \bar{V}_{+}, x-X_{j} \in \bar{V}_{+} \quad \text { for all } h, j
$$

and similarly for $r(X, \ldots)$.

d) The mass shell restrictions

$\tilde{r}_{M S}\left(\ldots, \boldsymbol{Q}_{1}, \ldots, \boldsymbol{Q}_{\alpha},-\boldsymbol{Q}_{\alpha+1}, \ldots,-\boldsymbol{Q}_{\beta}, \boldsymbol{q}_{1}, \ldots, \boldsymbol{q}_{\gamma},-\boldsymbol{q}_{\gamma+1}, \ldots,-\boldsymbol{q}_{\delta}\right)$

$=\left.\tilde{r}\left(\ldots, \bar{Q}_{1}, \ldots, \bar{Q}_{\alpha},-\bar{Q}_{\alpha+1}, \ldots,-\bar{Q}_{\beta}, \bar{q}_{1}, \ldots, \bar{q}_{\gamma},-\bar{q}_{\gamma+1}, \ldots,-\bar{q}_{\delta}\right)\right|_{Q_{J_{0}}=\omega\left(\boldsymbol{Q}_{j}\right), q_{h_{0}}=\omega\left(\boldsymbol{q}_{h}\right)}$

of the r-functions in p-space exist in the sense explained in $E$ and are defined on test functions of the form

$$
\varphi(\ldots) \psi_{1}\left(\boldsymbol{Q}_{1}, \ldots, \boldsymbol{Q}_{\alpha}, \boldsymbol{q}_{1}, \ldots, \boldsymbol{q}_{\gamma}\right) \psi_{2}\left(\boldsymbol{Q}_{\alpha+1}, \ldots, \boldsymbol{Q}_{\beta}, \boldsymbol{q}_{\gamma+1}, \ldots, \boldsymbol{q}_{\delta}\right),
$$

with $\varphi \in \mathscr{S}, \varphi_{1} \in L_{2}^{\alpha, \gamma}, \varphi_{2} \in L_{2}^{\beta-\alpha, \delta-\gamma}$.

Then there exists an asymptotically complete Wightman theory of two scalar, hermitian, local fields with masses $M$ and $m$ such that their retarded functions are the given ones up to the physically irrelevant ambiguities inherent in the definition of the R-product.

In (2.5) we have used the notation

$$
\omega(\boldsymbol{Q})=\sqrt{\boldsymbol{Q}^{2}+M^{2}}, \quad \omega(\boldsymbol{q})=\sqrt{\boldsymbol{q}^{2}+m^{2}} .
$$


$L_{2}^{\alpha, \gamma}$ is the space of square integrable functions with respect to the measure

$$
\prod_{1}^{\alpha} \frac{d^{3} Q_{j}}{2 \omega\left(\boldsymbol{Q}_{j}\right)} \prod_{1}^{\gamma} \frac{d^{3} q_{h}}{2 \omega\left(\boldsymbol{q}_{h}\right)} .
$$

Comparison of this formulation with Theorem 6 of $E$ shows that condition d) as stated here is somewhat too strong. Field theories may exist in which it is not satisfied in this form. The exact form is, however, quite complicated and we shall use the stronger one given here for the sake of simplicity. Our procedure could easily be adapted to the exact form.

It will be convenient to write (2.3) in a different way by amputating all $r$-functions with respect to the $B$-variables $x, y, x_{h}$. This can be done by putting bars over all those variables in (2.3). The original $r$-functions can be recovered from the amputated ones by

$$
\begin{aligned}
& r\left(X_{1}, \ldots, X_{a}, x_{1}, \ldots, x_{b}\right)=\int \prod_{1}^{b}\left\{d u_{h} \Delta_{R}\left(u_{h}-x_{h}\right)\right\} r\left(X_{1}, \ldots, X_{a}, \bar{u}_{1}, \ldots, \bar{u}_{b}\right), \\
& r\left(x_{1}, \ldots, x_{b}, X_{1}, \ldots, X_{a}\right) \\
& \quad=\int d u_{1} \Delta_{R}\left(x_{1}-u_{1}\right) \prod_{2}^{b}\left\{d u_{h} \Delta_{R}\left(u_{h}-x_{h}\right)\right\} r\left(\bar{u}_{1}, \ldots, \bar{u}_{b}, X_{1}, \ldots, X_{a}\right) .
\end{aligned}
$$

It is easy to see what becomes of the subsidiary conditions of Theorem 1 under the process of amputation.

The amputated system (2.3) together with the subsidiary conditions is equivalent to the original assumptions, if we add yet another subsidiary condition, to wit:

$$
\tilde{r}(\bar{p}, \bar{q})=\delta^{4}(p+q)\left\{-\frac{1}{2 \pi}\left(q^{2}-m^{2}\right)+\left(q^{2}-m^{2}\right)^{2} F\left(q^{2}\right)\right\},
$$

with $F$ analytic in a neighbourhood of $q^{2}=m^{2}$. This is the Kälén renormalization condition. It is a consequence of the unamputated form of (2.3) but is lost under amputation and has therefore to be demanded separately.

Henceforth we shall deal exclusively with amputated photon variables. We can therefore drop the amputation bars over photon variables, with the understanding that such variables are always amputated when they appear as arguments of the field $B$ or of a retarded or time-ordered product of $B$ 's.

The amputation of (2.3) has the consequence that the terms on the right-hand side containing the photon two-point function $r(x, y)$ vanish, i.e., these functions occur only in the $a=0, b=2$ case on the left. 
The following abbreviated notation will be convenient: we define

$$
\begin{aligned}
& r\left(x, x_{L}, X_{L} \rightarrow\right) r\left(y, x_{R}, X_{R} \leftarrow\right) \\
& \equiv \sum_{\alpha=0}^{\infty} \frac{i^{\alpha}}{\alpha !} \prod_{1}^{\alpha}\left\{d U_{j} d V_{j} \Delta_{+}\left(U_{j}-V_{j}\right)\right\} r\left(x, x_{L}, X_{L}, \bar{U}_{1}, \ldots, \bar{U}_{\alpha}\right) \\
& \quad \cdot r\left(y, x_{R}, X_{R}, \bar{V}_{1}, \ldots, \bar{V}_{\alpha}\right)
\end{aligned}
$$

where $x_{L}$, etc., are sets of variables and the distinguished variables $x, y$ might also be hadron variables. (2.3) can then be written:

$$
\begin{aligned}
r(x, y, \ldots) & -r(y, x, \ldots) \\
= & -i \sum_{L} \sum_{\beta=0}^{\infty} \frac{i^{\beta}}{\beta !}\left[\int \prod_{1}^{\beta}\left\{d u_{h} d v_{h} \Delta_{+}\left(u_{h}-v_{h}\right)\right\}\right. \\
& \left.\quad r\left(x, x_{L}, X_{L}, \bar{u}_{1}, \ldots, \bar{u}_{\beta} \rightarrow\right) r\left(y, x_{R}, X_{R}, \bar{v}_{1}, \ldots, \bar{v}_{\beta} \leftarrow\right)-(x \leftrightarrow y)\right] .
\end{aligned}
$$

We have yet to reformulate the conditions of Theorem 1 in terms of the time-ordered functions $\tau\left(X_{1}, \ldots, X_{a}, x_{1}, \ldots, x_{b}\right)$. We shall use a definition of $\tau$ which differs from the one adopted in $E$ by a factor $(-i)^{a+b-1}$. The defining equation of the $T$-products (Eq. (19) of $E$ ) becomes then

$$
R\left(X_{1}, \ldots, x_{b}\right)=T\left(X_{1}, \ldots, x_{b}\right)+i \sum_{L} T^{*}\left(X_{L}, x_{L}\right) T\left(X_{1}, X_{R}, x_{R}\right) .
$$

Here the sum extends over all partitions of $\left\{X_{2}, \ldots, X_{a}, x_{1}, \ldots, x_{b}\right\}$ into two complementary subsets $\left\{X_{L}, x_{L}\right\}$ and $\left\{X_{R}, x_{R}\right\}$, the second of which may be empty, but not the first.

We remember that the retarded and the truncated (=connected) time-ordered functions are in $p$-space boundary values of the same analytic function [14-16]. Let

$$
\begin{aligned}
\tilde{r}\left(P_{1}, \ldots, P_{a}, p_{1}, \ldots, p_{b}\right)= & (2 \pi)^{-\frac{s}{2}(a+b)} \int d X_{j} d x_{h} \\
& \cdot \exp \left\{i\left[\sum P_{j} X_{j}+\sum p_{h} x_{h}\right]\right\} r\left(X_{1}, \ldots, x_{b}\right) \\
= & \delta^{4}\left(\sum P_{j}+\sum p_{h}\right) \hat{r}\left(P_{1}, \ldots, p_{b}\right) .
\end{aligned}
$$

$\hat{r}$ is a distribution on the plane $\sum P_{j}+\sum p_{h}=0 . \hat{r}\left(p_{1}, \ldots, P_{a}\right)$ is defined analogously.

Introduce the complex four-vectors $K_{j}=P_{j}+i Q_{j}, k_{h}=p_{h}+i q_{h}$. Let $S$ be the subset of the plane $\sum K_{j}+\sum k_{h}=0$ defined by the condition that for any proper subs̉et $I \subset\left\{K_{1}, \ldots, k_{b}\right\}$ we have

$$
\left(\sum_{I} Q_{j}+\sum_{I} q_{h}\right)^{2}>0, \quad \text { or } \quad \sum_{I} Q_{j}+\sum_{I} q_{h}=0 \text { and }\left(\sum_{I} P_{j}+\sum_{I} p_{h}\right)^{2}<m^{2} \text {. }
$$


Then there exists a function $H\left(K_{1}, \ldots, K_{a}, k_{1}, \ldots, k_{b}\right)$ analytic in $S$, such that

$$
\begin{aligned}
& \hat{r}\left(P_{1}, \ldots, p_{b}\right)=\lim _{Q_{j}, q_{h} \rightarrow 0} H\left(K_{1}, \ldots, k_{b}\right) \\
& \hat{r}\left(p_{1}, \ldots, P_{a}\right)=\lim _{Q_{1}, q_{h} \rightarrow 0} H\left(K_{1}, \ldots, k_{b}\right),
\end{aligned}
$$

where the limit must be taken from the set $Q_{2}, \ldots, Q_{a}, q_{1}, \ldots, q_{b} \in V_{-}$ in the first case, from $Q_{1}, \ldots, Q_{a}, q_{2}, \ldots, q_{b} \in V_{-}$in the second case.

Let $T \subset S$ be the set defined by the conditions

$$
\sum_{I} Q_{J}+\sum_{I} q_{h} \in\left\{\begin{array}{l}
V_{+} \\
V_{-}
\end{array}\right\} \text {if }\left(\sum_{I} P_{j}+\sum_{I} p_{h}\right)_{0}\left\{\begin{array}{l}
> \\
<
\end{array}\right\} 0 .
$$

If $\left(\sum_{I} P_{j}+\sum_{I} p_{h}\right)_{0}=0$, either half cone is admissible for the corresponding $q$-sum.

Let $\tau^{T}$ be the truncated $\tau$-function and define

$$
\tilde{\tau}^{T}\left(P_{1}, \ldots, p_{b}\right)=\delta^{4}\left(\sum P_{j}+\sum p_{h}\right) \hat{\tau}\left(P_{1}, \ldots, p_{b}\right) .
$$

Then $\hat{\tau}$ is again a boundary value of $H$ in the sense of (2.14), where the limit has to be taken from $T$.

The distributions $\tilde{\tau}^{T}\left(P_{1}, \ldots, p_{b}\right)$ have the following linear properties:

$\alpha) \tilde{\tau}^{T}$ is Poincaré-invariant, symmetrical under permutations of the arguments and satisfies the PCT relation

$$
\tilde{\tau}^{T}\left(P_{1}, \ldots, p_{b}\right)=\tilde{\tau}^{T}\left(-P_{1}, \ldots,-p_{b}\right) .
$$

$\beta$ ) The mass shell restrictions of $\tilde{\tau}^{T}$ exist in the same way as those of $\tilde{r}$ defined in Theorem 1 .

Furthermore, the $\tau^{T}$ satisfy the completeness equations (better known under the name unitarity equations) [8]:

$$
\begin{aligned}
& \tilde{\tau}^{T}\left(P_{1}, \ldots, p_{b}\right)-\tilde{\tau}^{T^{*}}\left(P_{1}, \ldots, p_{b}\right) \\
& =-i \sum_{L} \sum_{\beta=0}^{\infty} \frac{(2 \pi)^{2 \beta}}{\beta !}\left[\int \prod_{1}^{\beta}\left\{d w_{h} \delta_{+}\left(w_{h}\right)\right\} \tilde{\tau}^{*}\left(P_{L}, p_{L},-w_{j} \rightarrow\right) \tilde{\tau}\left(P_{R}, p_{R}, w_{j} \leftarrow\right)\right]^{T} .
\end{aligned}
$$

The sum $\sum_{L}$ extends over all partitions of $\left\{P_{1}, \ldots, p_{b}\right\}$ into two nonempty sets $L$ and $R$. The definitions

$$
\begin{aligned}
& \tilde{\tau}^{*}\left(P_{L}, p_{L} \rightarrow\right) \tilde{\tau}\left(P_{R}, p_{R} \leftarrow\right) \\
& =\sum_{\alpha=0}^{\infty} \frac{(2 \pi)^{2 \alpha}}{\alpha !} \cdot \int \prod_{1}^{\alpha}\left\{d W_{j} \delta_{+}\left(W_{j}\right)\right\} \tilde{\tau}^{*}\left(P_{L}, p_{L},-\bar{W}_{h}\right) \tilde{\tau}\left(P_{R}, p_{R}, \bar{W}_{h}\right), \\
& \delta_{+}(W)=\theta\left(W_{0}\right) \delta\left(W^{2}-M^{2}\right), \quad \delta_{+}(w)=\theta\left(w_{0}\right) \delta\left(w^{2}-m^{2}\right)
\end{aligned}
$$


have been used. The truncated part of a complicated expression like the right-hand side of (2.18) is defined as that part which does not contain any $\delta^{4}$-functions besides the $\delta^{4}\left(\sum P_{j}+\sum p_{h}\right)$ expressing overall momentum conservation.

Condition (2.8) holds for $\tilde{\tau}$ in exactly the same way as for $\tilde{r}$.

These conditions on $\tilde{\tau}^{T}$ are equivalent to the conditions of Theorem 1 , i.e., we have:

Theorem 2. Let $\hat{\tau}\left(P_{1}, \ldots, p_{b}\right)$ be boundary values of functions $H$ analytic in $S$, the boundary being attained from $T$. Let $\tilde{\tau}^{T}$ as defined by (2.16) satisfy Eqs. (2.18) and enjoy the properties $\alpha$ ) and $\beta$ ) above as well as (2.8).

Then the functions $\tilde{r}$ defined by (2.14) and (2.12) satisfy the assumptions of Theorem 1.

The proof of this theorem proceeds by analytic continuation in $p$ space and will not be given here. We think that the theorem is plausible enough in itself.

\section{Perturbation Theory: Generalities}

We assume that the fields $A$ and $B$ are coupled through an interaction with a small coupling constant $e$. We develop $r$ with respect to $e$ :

$$
r\left(X_{1}, \ldots, x_{b}\right)=\sum_{\sigma=0}^{\infty} e^{\sigma} r_{\sigma}\left(X_{1}, \ldots, x_{b}\right)
$$

and wish to determine the $r_{\sigma}$ such that the conditions of Theorem 1 are satisfied in every order $\sigma$. (Actually, we shall only proceed as far as $\sigma=2$.)

In 0 th order we assume $B$ to be free, i.e., we demand

$$
\begin{aligned}
r_{0}\left(X_{1}, \ldots, x_{b}\right) & =r_{0}\left(x_{1}, \ldots, X_{a}\right)=0 \text { for } a \neq 0, b \neq 0, \\
r_{0}\left(x_{1}, \ldots, x_{b}\right) & =0 \text { for } b>2, \\
r_{0}\left(x_{1}, x_{2}\right) & =\left(\square_{x_{1}}+m^{2}\right) \delta^{4}\left(x_{1}-x_{2}\right) .
\end{aligned}
$$

The $r_{0}\left(X_{1}, \ldots, X_{a}\right)$ shall be the $r$-functions of a self-interacting field $A_{0}$. They have then all the properties stated in Theorem 1, now formulated for the case of one field only. In particular we have

$$
\begin{aligned}
r_{0}\left(X, Y, X_{1}, \ldots, X_{a}\right)-r_{0}\left(Y, X, X_{1}, \ldots, X_{a}\right) \\
=-i \sum_{L}\left\{r_{0}\left(X, X_{L} \rightarrow\right) r_{0}\left(Y, X_{R} \leftarrow\right)-(X \leftrightarrow Y)\right\} .
\end{aligned}
$$


Inserting (3.1) into (2.10) we obtain as terms of order $\sigma$ :

$$
\begin{aligned}
r_{\sigma}(X, Y, \ldots)-r_{\sigma}(Y, X, \ldots) & =-i \sum_{L} \sum_{\beta=0}^{\infty} \frac{i^{\beta}}{\beta !} \sum_{v=0}^{\sigma}\left[\int \prod_{1}^{\beta}\left\{d u_{h} d v_{h} \Delta_{+}\left(u_{h}-v_{h}\right)\right\}\right. \\
& \left.\cdot r_{\sigma-v}\left(X, \ldots, \bar{u}_{\beta} \rightarrow\right) r_{v}\left(Y, \ldots, \bar{v}_{\beta} \leftarrow\right)-(X \leftrightarrow Y)\right] .
\end{aligned}
$$

These equations we wish to solve by induction with respect to $\sigma$. Assume that the $r_{v}$ with $v<\sigma$ are known. Then (3.4) is a system of linear equations for the unknown quantities $r_{\sigma}$. It has been noted in Section II that the right-hand side of (2.10) does not contain the two-point $B$-field function. Due to this fact and to (3.2), the unknown $r_{\sigma}$ 's appear in the right-hand side of (3.4) only in the terms

$$
\begin{aligned}
-i \sum_{L}\left\{r _ { \sigma } \left(X, X_{L},\right.\right. & \left.x_{1}, \ldots, x_{b} \rightarrow\right) r_{0}\left(Y, X_{R} \leftarrow\right) \\
& \left.+r_{0}\left(X, X_{L} \rightarrow\right) r_{\sigma}\left(Y, X_{R}, x_{1}, \ldots, x_{b} \leftarrow\right)-(X \leftrightarrow Y)\right\} .
\end{aligned}
$$

All the photon variables appear always in the same factor. This has the important consequence that Eqs. (3.4) decouple with respect to the number $b$ of external photon variables.

\section{Perturbation Theory : First Order}

For $\sigma=1$ Eqs. (3.4) are homogeneous in the unknown functions $r_{1}$. It has already been noted that they decouple with respect to $b$ :

$$
\begin{aligned}
r_{1}\left(X, Y, \ldots, X_{j}, \ldots, x_{h}, \ldots\right) & -r_{1}\left(Y, X, \ldots, X_{j}, \ldots, x_{h}, \ldots\right) \\
= & -i \sum_{L}\left\{r_{1}\left(X, X_{L}, \ldots, x_{h}, \ldots \rightarrow\right) r_{0}\left(Y, X_{R} \leftarrow\right)\right. \\
& \left.+r_{0}\left(X, X_{L} \rightarrow\right) r_{1}\left(Y, X_{R}, \ldots, x_{h}, \ldots \leftarrow\right)-(X \leftrightarrow Y)\right\} .
\end{aligned}
$$

An obvious solution (for a given $b$ ) is $r_{1}\left(X_{1}, \ldots\right)=r_{1}\left(x_{1}, \ldots\right)=0$ for all $a$. In the electromagnetic case this is true for all $b \neq 1$. Only this case will be considered further:

$r_{1}\left(X_{1}, \ldots, X_{a}, x_{1}, \ldots, x_{b}\right)=r_{1}\left(x_{1}, \ldots, x_{b}, X_{1}, \ldots, X_{a}\right)=0$ for $b \neq 1$.

This condition corresponds to an interaction Lagrangian which is linear in $B$.

The equations to be solved in the case $b=1$ are (4.1) with only one photon variable, called $x$, present.

We note that no summation over intermediary states containing photons occurs in (4.1). Hence we can consider (4.1) as an equation referring to $\mathfrak{S}_{A}$ only. We obtain: 
Lemma 1. Let $r_{1}$ be a solution of (4.1) satisfying conditions b)-d) of Theorem 1.

Then

$$
J(x)=\sum_{l=1}^{\infty} \frac{1}{l !} \int d U_{1} \ldots d U_{l} r_{1}\left(x, \bar{U}_{1}, \ldots, \bar{U}_{l}\right): A^{\mathrm{in}}\left(U_{1}\right) \ldots A^{\mathrm{in}}\left(U_{l}\right):
$$

converges strongly on all states in $\mathfrak{S}_{A}$ with a finite number of incoming particles. $J(x)$ is a scalar, hermitian field which is local with respect to $A_{0}(X)$.

The proof of this lemma is essentially an adaptation of the proof of Theorem 5 of $E$. It is obvious that the matrix elements of the series (4.3) between in-states with a finite number of particles converge, since in this case only a finite number of terms are different from zero. In order to prove strong convergence we must look ahead to second-order perturbation theory. There, expressions of the type

$$
r_{1}\left(x, X_{1}, \ldots, X_{m} \rightarrow\right) r_{1}\left(y, Y_{1}, \ldots, Y_{n} \leftarrow\right)
$$

will occur. These expressions must exist, or else our perturbation procedure will break down already in second order. We shall therefore assume the existence of (4.4), whence the strong convergence of (4.3) can be proved as in $E$.

It is obvious that $J$ is scalar and hermitian. Using

$$
A_{0}(X)=\sum_{l=1}^{\infty} \frac{1}{l !} \int d U_{1} \ldots d U_{l} r_{0}\left(X, \bar{U}_{1}, \ldots, \bar{U}_{l}\right): A^{\mathrm{in}}\left(U_{1}\right) \ldots A^{\mathrm{in}}\left(U_{l}\right):
$$

we can calculate the commutator $\left[J(x), A_{0}(X)\right]$. By reducing products of Wick products to sums of Wick products in the customary way and making use of (4.1) we find

$$
\begin{aligned}
& {\left[J(x), A_{0}(X)\right]} \\
& \begin{array}{r}
=\sum_{l=1}^{\infty} \frac{1}{l !} \int d U_{1} \ldots d U_{l}\left[r_{1}\left(x, X, \bar{U}_{1}, \ldots, \bar{U}_{l}\right)-r_{1}\left(X, x, \bar{U}_{1}, \ldots, \bar{U}_{l}\right)\right] \\
: A^{\text {in }}\left(U_{1}\right) \ldots A^{\text {in }}\left(U_{l}\right) .
\end{array}
\end{aligned}
$$

from which the relative locality of $J$ follows because of the support conditions imposed on $r_{1}$. Due to a theorem of Borchers [17] $J$ is then also local relative to itself.

Furthermore we want to have $r_{1}(x)=0$, which leads to

$$
(\Omega, J(x) \Omega)=0 .
$$

On the other hand, it is clear that any scalar hermitian field $J$ in $\mathfrak{S}_{A}$, local relative to $A_{0}$, and with a vanishing vacuum expectation value, 
will produce a solution of (4.1) via the definition

$$
\begin{aligned}
& r_{1}\left(x, X_{1}, \ldots, X_{a}\right)=\left(\Omega, R\left[J(x) A_{0}\left(X_{1}\right) \ldots A_{0}\left(X_{a}\right)\right] \Omega\right) \\
& r_{1}\left(X_{1}, \ldots, X_{a}, x\right)=\left(\Omega, R\left[A_{0}\left(X_{1}\right) \ldots A_{0}\left(X_{a}\right) J(x)\right] \Omega\right),
\end{aligned}
$$

the $R$-product being defined as in $E$. Hence:

Theorem 3. The general solution of (4.1) in the case $b=1$, satisfying the required subsidiary conditions, is given by (4.8), where $J(x)$ is an arbitrary scalar, hermitian, local (relative to $A_{0}$ ) field in $\mathfrak{H}_{A}$ with a vanishing vacuum expectation value.

The choice of this field $J$ specifies the interaction, i.e., $J$ replaces the interaction Lagrangian (or Hamiltonian) of the conventional formulation of perturbation theory.

How such an operator can be characterized explicitly is no known at present. The ansatz

$$
J(x)=\sum c_{v}\left[A_{0}(x)\right]^{v}
$$

(plus terms containing derivatives of $A_{0}$ ) does of course formally satisfy our requirements. It has been shown by Zimmermann [18] how expressions of the type (4.9) can be defined rigorously, if perturbation theory is used with respect to the strong interactions also. It is not known whether this method of definition can be applied in an exact theory, much less whether all possible $J$ could be obtained in this way. We shall leave this question open and shall simply assume that an operator $J(x)$ is given somehow.

The statement that $J(x)$ defines the interaction has yet to be qualified somewhat because the definition of the $R$-product contains ambiguities. If $J$ is given, then $r_{1}\left(x, \bar{X}_{1}, \ldots, \bar{X}_{a}\right)$ and $r_{1}\left(\bar{X}_{1}, \ldots, \bar{X}_{a}, x\right)$ are defined unambiguously if all the variables are put on their respective mass shells. That means that the first order $S$-matrix elements are unambiguously defined. However, $r_{1}\left(X_{1}, \bar{X}_{2}, \ldots, \bar{X}_{a}, x\right)$ may contain ambiguities, i.e., the first-order term $A_{1}$ of the hadron field:

$$
\begin{aligned}
A_{1}(X)=\sum_{l=1}^{\infty} \frac{1}{l !} \int d U_{1} \ldots d U_{l} d u r_{1}\left(X, \bar{U}_{1}, \ldots, \bar{U}_{l}, u\right) & \\
& : A^{\text {in }}\left(U_{1}\right) \ldots A^{\text {in }}\left(U_{l}\right) B^{\text {in }}(u):
\end{aligned}
$$

will contain ambiguities which will influence the $S$-matrix in higher orders. The interaction is therefore specified not by $J$ alone but by $J$ together with a particular choice of the $R$-product in (4.8). The ambiguity can be reduced by imposing on $r_{1}$ conditions about the behaviour at large momenta (or at small distances in $x$-space, which amounts to the 
same) of the type used in Ref. [9]. Loosely speaking, these conditions demand that $\tilde{r}$ behave at infinity as well as possible. Under favourable circumstances this condition may actually remove the ambiguity, but this question cannot be discussed without an explicit knowledge of $r_{0}$ and $J$. Luckily, it will turn out that this indeterminacy does not influence the second-order mass shift.

For later reference we shall briefly consider the non-trivial solutions of (4.1) in the case $b=2$. In this case one of those Eqs. (4.1) which have not been written down explicitly reads

$$
r_{1}\left(x, y, X_{1}, \ldots, X_{a}\right)-r_{1}\left(y, x, X_{1}, \ldots, X_{a}\right)=0,
$$

whence we see immediately that $r_{1}\left(x, y, X_{1}, \ldots\right)$ has its support in $x=y$. A similar argument as in the case $b=1$ yields the result:

Theorem 4. The general solution of (4.1) in the case $b=2$, with the correct linear properties, is

$$
r_{1}\left(x, y, X_{1}, \ldots, X_{a}\right)=\sum_{D} D \delta^{4}(x-y)\left(\Omega, R\left[J^{D}(y) A_{0}\left(X_{1}\right) \ldots A_{0}\left(X_{a}\right)\right] \Omega\right)
$$

and similarly for $r_{1}\left(X_{1}, \ldots, X_{a}, x, y\right)$, where the sum extends over a finite number of derivatives (with respect to $x$ ) $D$, and the $J^{D}$ are local hermitian field in $\mathfrak{S}_{A}$ with covariance properties designed to make the D-sum invariant.

The same remarks on the ambiguities in the definition of the $R$-product as given after Theorem 3 apply here.

\section{Perturbation Theory: Second Order}

If $b$ is not equal to 0 or 2, Eqs. (3.4) for $\sigma=2$ again reduce to the homogeneous form (4.1) and are solved by

$r_{2}\left(X_{1}, \ldots, X_{a}, x_{1}, \ldots, x_{b}\right)=r_{2}\left(x_{1}, \ldots, x_{b}, X_{1}, \ldots, X_{a}\right)=0$ for $b \neq 0$ or 2.

This is the most regular solution and will therefore be assumed to be the correct one.

Case $b=2$. In this case we have to solve the equations

$$
\begin{aligned}
r_{2}\left(X, Y, \ldots, X_{j}, \ldots,\right. & \left.x_{1}, x_{2}\right)-r_{2}\left(Y, X, \ldots, X_{j}, \ldots, x_{1}, x_{2}\right) \\
& +i \sum_{L}\left\{r_{2}\left(X, X_{L}, x_{1}, x_{2} \rightarrow\right) r_{0}\left(Y, X_{R} \leftarrow\right)\right. \\
& \left.+r_{0}\left(X, X_{L} \rightarrow\right) r_{2}\left(Y, X_{R}, x_{1}, x_{2} \leftarrow\right)-(X \leftrightarrow Y)\right\} \\
= & -i \sum_{L}\left\{r_{1}\left(X, X_{L}, x_{1} \rightarrow\right) r_{1}\left(Y, X_{R}, x_{2} \leftarrow\right)\right. \\
& \left.+r_{1}\left(X, X_{L}, x_{2} \rightarrow\right) r_{1}\left(Y, X_{R}, x_{1} \leftarrow\right)-(X \leftrightarrow Y)\right\}
\end{aligned}
$$


and similar equations in which one or both of the distinguished variables $X, Y$ are replaced by an $x_{h}$. They can again be considered as equations in $\mathfrak{G}_{A}$ and have the obvious solution

$$
\begin{aligned}
& r_{2}\left(X_{1}, \ldots, X_{a}, x_{1}, x_{2}\right)=\left(\Omega, R\left[A_{0}\left(X_{1}\right) \ldots A_{0}\left(X_{a}\right) J\left(x_{1}\right) J\left(x_{2}\right)\right] \Omega\right), \\
& r_{2}\left(x_{1}, x_{2}, X_{1}, \ldots, X_{a}\right)=\left(\Omega, R\left[J\left(x_{1}\right) J\left(x_{2}\right) A_{0}\left(X_{1}\right) \ldots A_{0}\left(X_{a}\right)\right] \Omega\right)
\end{aligned}
$$

The general solution of (5.2) is given by (5.3) plus the general solution of the corresponding homogeneous equation as described in Theorem 4. The ambiguity can again be decreased by demanding optimal behaviour for small distances. In favourable cases this may lead to a unique solution. We shall assume this to be the case at least for $a=2$ since only then our derivation of a formula for the mass shift will be convincing.

If this procedure is applied to realistic electrodynamics, the requirements of gauge invariance will have to be taken into account. They may enforce the addition of a homogeneous term to (5.3). Such an addition may also be necessary in our model for the two-point case $(a=0)$ in order to satisfy condition (2.8). We shall not discuss the point any further since in electrodynamics this condition will presumably be satisfied automatically due to gauge invariance, if our experiences gained in Lagrangian field theory are at all to be trusted.

Case $b=0$. Here it is convenient to work with the $\tau$-functions instead of the $r$-functions, i.e., to make use of Theorem 2. We have then to solve the equations

$$
\begin{array}{r}
\tilde{\tau}_{2}^{T}\left(P_{1}, \ldots, P_{a}\right)-\tilde{\tau}_{2}^{T^{*}}\left(P_{1}, \ldots, P_{a}\right)+i \sum_{L}\left[\tilde{\tau}_{2}^{*}\left(P_{L} \rightarrow\right) \tilde{\tau}_{0}\left(P_{R} \leftarrow\right)+\tilde{\tau}_{0}^{*}\left(P_{L} \rightarrow\right) \tilde{\tau}_{2}\left(P_{R} \leftarrow\right)\right]^{T} \\
=-2 \pi i \sum_{L}\left[\int d w \delta_{+}(w) \tilde{\tau}_{1}^{*}\left(P_{L},-w \rightarrow\right) \tilde{\tau}_{1}\left(P_{R}, w \leftarrow\right)\right]^{T} .
\end{array}
$$

Remember that both $P_{L}$ and $P_{R}$ must be non-empty.

We shall first try to solve (5.4) with the ansatz

$$
\tilde{\tau}_{2}^{T}\left(P_{1}, \ldots, P_{a}\right)=\frac{i}{2}\left[\int d w \frac{\tilde{\tau}_{2}\left(P_{1}, \ldots, P_{a}, w,-w\right)}{w^{2}-m^{2}+i \varepsilon}\right]^{T}
$$

suggested by the Feynman rules. Here $\tilde{\tau}_{2}^{T}\left(P_{1}, \ldots, P_{a}, p_{1}, p_{2}\right)$ is obtained from $\tilde{r}_{2}\left(P_{1}, \ldots, p_{2}\right)$ by analytic continuation as explained in Section II.

Consider the holomorphic function $H_{2}\left(K_{1}, \ldots, K_{a}, k_{1}, k_{2}\right)$ of which both $\hat{r}_{2}\left(P_{1}, \ldots, p_{2}\right)$ and $\hat{\tau}_{2}\left(P_{1}, \ldots, p_{2}\right)$ are boundary values. Fix the values of the variables $K_{j}$ such that their space parts are real: $\boldsymbol{K}_{j}=\boldsymbol{P}_{j}$ and that $\left(\sum_{I} Q_{i}\right)_{0} \neq 0$ for all proper subsets $I$ of $(1, \ldots, a)$. Then $H_{2}\left(\ldots, K_{a}, w,-w\right)$ is, for real $\boldsymbol{w}$, analytic in the full $w_{0}$-plane with the exception of the cuts

$$
w_{0}= \pm\left(\sum_{I} K_{J}\right)_{0} \pm\left[M_{I}\left(\boldsymbol{P}_{j}, w\right)+\varrho\right]
$$




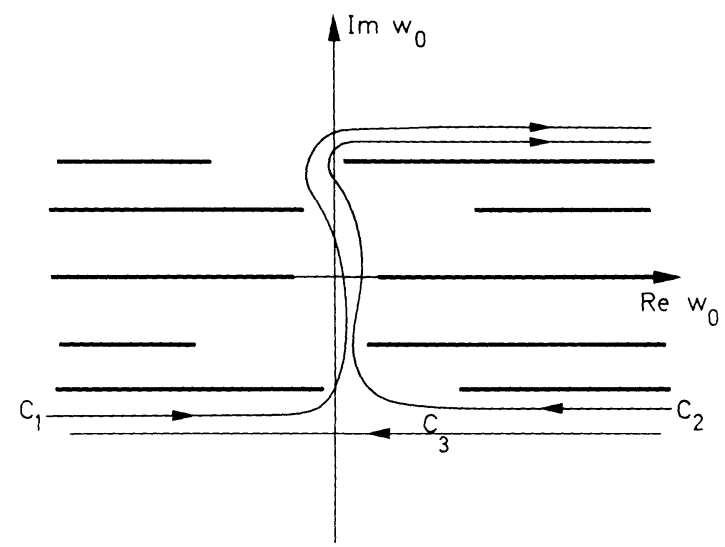

Fig. 1. The integration paths $C_{1}, C_{2}$ and $C_{3}$. The $w_{0}$-plane is shown with some of the cuts (5.6) marked by bold lines

for all $I$, including the empty set. Here $M_{I}$ is a real function of its arguments with $M_{I} \geqq m$, and $\varrho \geqq 0$ is parametrizing the cut. The two sign alternatives are independent of each other.

Under the above restrictions for $K_{j}$ we obtain from (5.5)

$$
\begin{aligned}
H_{2}\left(K_{1}, \ldots, K_{a}\right)=\frac{i}{2}\left[\int d^{3} \boldsymbol{w} \int_{C_{1}} d w_{0} \frac{H_{2}\left(K_{1}, \ldots, K_{a}, w,-w\right)}{w^{2}-m^{2}}\right. \\
\left.\quad+\left.2 \pi \sum_{L} \frac{H_{1}\left(K_{L},-k\right) H_{1}\left(K_{R}, k\right)}{k^{2}-m^{2}}\right|_{k=\sum_{L} K_{J}}\right] .
\end{aligned}
$$

The integration contour $C_{1}$ is given in Fig. 1 . The $d^{3} w$ integration runs over real $\boldsymbol{w}$.

This $H_{2}$ can easily be shown to have the correct analyticity properties, provided that the integral (5.7) exists at all. Since the integrand is on $C_{1}$ analytic in $w$, existence depends solely on the behaviour at infinity. We shall assume that the integral (5.7), and therefore (5.5), exists and that the mass shell restrictions of $\tilde{\tau}_{2}^{T}$ exist as explained in Section II (property $\beta$ ). Moreover, we shall assume that the $w$-integration in (5.5) can be interchanged with the $P_{j}$-integrations over test functions.

Note that (5.7) can exist for at most one solution $r_{2}$ of (5.2). The difference between two solutions is, according to Theorem 4, a polynomial in $w$ and will therefore give a divergent contribution to (5.7). The one good $\tilde{r}_{2}$ (if it exists) will be distinguished by its behaviour at infinity, i.e., it will be the special solution chosen above. If such a distinguished solution does not exist (i.e., if our regularity requirements do not 
fix the solution of (5.2) uniquely), then most likely no $H_{2}(\ldots, w,-w)$ will give a convergent integral (5.7). This is the reason why we assumed the existence of a unique solution of (5.2) with optimal behaviour at infinity in $p$-space.

Invariance and symmetry of $\tilde{\tau}_{2}^{T}\left(P_{1}, \ldots, P_{a}\right)$ as given by (5.5) is obvious. The same is true for the relation

$$
\tilde{\tau}_{2}^{T}\left(\ldots, P_{j}, \ldots\right)=\tilde{\tau}_{2}^{T}\left(\ldots,-P_{j}, \ldots\right)
$$

once we havenoted that the corresponding relation holds for $\tilde{\tau}_{2}^{T}\left(\ldots, P_{a}, p_{1}, p_{2}\right)$ as a result of the reality of $r_{2}\left(\ldots, X_{a}, x_{1}, x_{2}\right)$.

The one-point function $\tilde{\tau}_{2}(P)$ vanishes automatically due to the pseudoscalarity of $A(X)$. (This automatic vanishing is true for realistic hadrons.) Otherwise we should have to obtain this desirable property by adding a constant to the field $A_{2}(X)$.

It remains to be seen whether (5.5) satisfies the completeness Eqs. (5.4). Inserting (5.5) into (5.4) and using

$$
\frac{1}{w^{2}-m^{2}+i \varepsilon}=\frac{\mathscr{P}}{w^{2}-m^{2}}-i \pi\left[\delta_{+}(w)+\delta_{+}(-w)\right],
$$

we obtain for the difference $\Delta$ of the two sides of (5.4)

$$
\begin{aligned}
\Delta= & \frac{i}{2}\left[\int d w \frac { \mathscr { P } } { w ^ { 2 } - m ^ { 2 } } \left\{\tilde{\tau}_{2}\left(P_{1}, \ldots, P_{a}, w,-w\right)+\tilde{\tau}_{2}^{*}\left(P_{1}, \ldots, P_{a}, w,-w\right)\right.\right. \\
& \left.\left.-i \sum_{L} \tilde{\tau}_{2}^{*}\left(P_{L}, w,-w \rightarrow\right) \tilde{\tau}_{0}\left(P_{R} \leftarrow\right)+i \sum_{L} \tilde{\tau}_{0}^{*}\left(P_{L} \rightarrow\right) \tilde{\tau}_{2}\left(P_{R}, w,-w \leftarrow\right)\right\}\right]^{T} \\
& +\pi\left[\int d w \delta _ { + } ( w ) \left\{\tilde{\tau}_{2}\left(P_{1}, \ldots, P_{a}, w,-w\right)-\tilde{\tau}_{2}^{*}\left(P_{1}, \ldots, P_{a}, w,-w\right)\right.\right. \\
& +i \sum_{L} \tilde{\tau}_{2}^{*}\left(P_{L}, w,-w \rightarrow\right) \tilde{\tau}_{0}\left(P_{R} \leftarrow\right)+i \sum_{L} \tilde{\tau}_{0}^{*}\left(P_{L} \rightarrow\right) \tilde{\tau}_{2}\left(P_{R}, w,-w \leftarrow\right) \\
& \left.\left.+2 i \sum_{L} \tilde{\tau}_{1}^{*}\left(P_{L},-w \rightarrow\right) \tilde{\tau}_{1}\left(P_{R}, w \leftarrow\right)\right\}\right]^{T} .
\end{aligned}
$$

We note that in the first term any odd function of $w$ can be added inside the curly bracket without changing the integral. With the help of the equation (derived from (2.11))

$$
\begin{aligned}
& \tilde{r}_{2}\left(w,-w, P_{1}, \ldots, P_{a}\right)=\tilde{\tau}_{2}^{T}\left(P_{1}, \ldots, P_{a}, w,-w\right)+i \sum_{L} \tilde{\tau}_{0}^{*}\left(P_{L} \rightarrow\right) \tilde{\tau}_{2}\left(P_{R}, w,-w \leftarrow\right) \\
& \quad+i \sum_{L} \tilde{\tau}_{1}^{*}\left(P_{L},-w \rightarrow\right) \tilde{\tau}_{1}\left(P_{R}, w \leftarrow\right)+i \tilde{\tau}_{1}^{*}(-w \rightarrow) \tilde{\tau}_{1}\left(P_{1}, \ldots, P_{a}, w \leftarrow\right) \\
& \quad+i \tilde{\tau}_{1}^{*}\left(P_{1}, \ldots, P_{a},-w \rightarrow\right) \tilde{\tau}_{1}(w \leftarrow) \\
& 11 \text { Commun math Phys, Vol. 15 }
\end{aligned}
$$


the first term of (5.9) can be transformed into

$i \int \frac{d w}{w^{2}-m^{2}-i \varepsilon w_{0}} \tilde{r}_{2}\left(-w, w, P_{1}, \ldots, P_{a}\right)$
$+i \pi\left[\int d w \delta_{+}(w) \sum_{L}\left\{\tilde{\tau}_{1}^{*}\left(P_{L}, w \rightarrow\right) \tilde{\tau}_{1}\left(P_{R},-w \leftarrow\right)-\tilde{\tau}_{1}^{*}\left(P_{L},-w \rightarrow\right) \tilde{\tau}_{1}\left(P_{R}, w \leftarrow\right)\right\}\right]^{T}$

whence

$$
\begin{aligned}
\Delta= & i \int \frac{d w}{w^{2}-m^{2}-i \varepsilon w_{0}} \tilde{r}_{2}(-w, w, \ldots)+\pi\left[\int d w \delta _ { + } ( w ) \left\{\tilde{\tau}_{2}\left(P_{1}, \ldots, w,-w\right)\right.\right. \\
& -\tilde{\tau}_{2}^{*}\left(P_{1}, \ldots, w,-w\right)+i \sum_{L} \tilde{\tau}_{2}^{*}\left(P_{L}, w,-w \rightarrow\right) \tilde{\tau}_{0}\left(P_{R} \leftarrow\right) \\
& +i \sum_{L} \tilde{\tau}_{0}^{*}\left(P_{L} \rightarrow\right) \tilde{\tau}_{2}\left(P_{R}, w,-w \leftarrow\right) \\
& \left.\left.+i \sum_{L} \tilde{\tau}_{1}^{*}\left(P_{L}, w \rightarrow\right) \tilde{\tau}_{1}\left(P_{R},-w \leftarrow\right)+i \sum_{L} \tilde{\tau}_{1}^{*}\left(P_{L},-w \rightarrow\right) \tilde{\tau}_{1}\left(P_{R}, w \leftarrow\right)\right\}\right]^{T} \\
= & i \int \frac{d w}{w^{2}-m^{2}-i \varepsilon w_{0}} \tilde{r}_{2}\left(-w, w, P_{1}, \ldots, P_{a}\right),
\end{aligned}
$$

the curly bracket vanishing on account of $\tilde{\tau}_{2}$ being a solution of the $\tau$ completeness equations corresponding to (5.2).

Thus the ansatz (5.5) solves the completeness equation only if the expression (5.11) vanishes. The integrand of (5.11) is analytic in $\operatorname{Im} w_{0}<0$ and the integration path runs along the lower rim of the real $w_{0}$-axis. The integral vanishes if this path can be closed by an infinite semicircle in the lower half plane, i.e., if $\tilde{r}_{2}(-w, w, \ldots)$ decreases rapidly enough for $w_{0} \rightarrow \infty$ in the lower half plane. Otherwise $\Delta \neq 0$, and (5.5) does not solve (5.4)! Note that the assumed existence of the integral (5.5) does not necessarily imply a strong decrease of $\tilde{r}_{2}$ (or $\tilde{\tau}_{2}^{T}$ ) at infinity. If $H_{2}$ shows an oscillatory behaviour along $C_{1}$, then (5.5) may converge conditionally and yet $\Delta$ may be different from zero.

The form of the remainder term (5.11) suggests that we might obtain a true solution by integrating in (5.7) along the path $C_{2}$ of Fig. 1 instead of $C_{1}$. We can achieve this by adding to (5.7) the integral along $C_{3}$ and this can be determined as follows.

Define a new kind of product of field operators $S$ by

$$
\begin{aligned}
R\left(x_{1}, x_{2},\right. & \left.X_{1}, \ldots, X_{a}\right)=S\left(x_{1}, x_{2}, X_{1}, \ldots, X_{a}\right) \\
& +i \sum_{L} T^{*}\left(X_{L}\right) S\left(x_{1}, x_{2}, X_{R}\right)+i T^{*}\left(X_{1}, \ldots, X_{a}\right) S\left(x_{1}, x_{2}\right),
\end{aligned}
$$

from which identity $S$ can be constructed recursively. Let

$$
\sigma\left(x_{1}, x_{2}, \ldots, X_{j}, \ldots\right)=\left(\Omega, S\left(x_{1}, x_{2}, \ldots, X_{j}, \ldots\right) \Omega\right) .
$$


$S$ is a cycle in the sense of Ruelle [14] taking an intermediate position between retarded and time-ordered products.

$\hat{\sigma}\left(p_{1}, p_{2}, P_{1}, \ldots, P_{a}\right)$, defined as in $(2.12)$, is again a boundary value of $H$, where the limit now has to be taken from the domain

$$
\begin{gathered}
\sum_{I} Q_{j} \in\left\{\begin{array}{l}
V_{+} \\
V_{-}
\end{array}\right\} \quad \text { if }\left(\sum_{I} P_{j}\right)_{0}\left\{\begin{array}{l}
> \\
<
\end{array}\right\} 0, \\
q_{2} \in V_{-}, q_{2}+\sum_{I} Q_{j} \in V_{-} \text {for all subsets } I \text { of }\{1, \ldots, a\} .
\end{gathered}
$$

The contribution of the $C_{3}$-integral to $\tilde{\tau}_{2}^{T}$ is

$$
-\frac{i}{2}\left[\int \frac{d w}{w^{2}-m^{2}-i \varepsilon w_{0}} \tilde{\sigma}_{2}\left(-w, w, P_{1}, \ldots, P_{a}\right)\right]^{T},
$$

so that our new ansatz is

$\tilde{\tau}_{2}^{T}\left(P_{1}, \ldots, P_{a}\right)=\frac{i}{2}\left[\int d w\left\{\frac{\hat{\tau}_{2}\left(P_{1}, \ldots, P_{a}, w,-w\right)}{w^{2}-m^{2}+i \varepsilon}-\frac{\tilde{\sigma}_{2}\left(-w, w, P_{1}, \ldots, P_{a}\right)}{w^{2}-m^{2}-i \varepsilon w_{0}}\right\}\right]^{T}$

which is to replace (5.5). The same assumptions about the existence of this expression will be made as earlier for (5.5). All the required linear properties are again satisfied and substitution of (5.15) into (5.4) will show that this equation is now exactly solved.

The general solution of (5.4) is obtained by adding the general homogeneous solution (whose form is unknown) to the particular solution (5.15). Again we assume that the solution (5.15) is distinguished by its optimal behaviour at infinity. Otherwise the freedom in the choice of the homogeneous addition would introduce undetermined parameters, in particular an undetermined mass renormalization, which would make a calculation of the mass shift from first principles impossible. This problem also occurs if the integral (5.15) does not converge for any $\tilde{\tau}_{2}^{T}(\ldots, w,-w) .(5.15)$ then still constitutes a formal solution from which a finite solution can be derived in the customary way by adding a suitable homogeneous solution with infinite coefficients. Indeterminate parameters are thereby necessarily introduced and the mass shift becomes a free parameter which cannot be calculated.

\section{Mass Shift}

In Section $\mathrm{V}$ we have assumed that the mass-shell restrictions of (5.15) exist as stated in Theorem 1. We shall now show that this is in fact not true. 
The one-particle structure of $\hat{\tau}_{2}\left(P_{1}, \ldots, P_{a}, p_{1}, p_{2}\right)$ (and of $\left.\hat{\sigma}_{2}\right)$ can be studied with the methods used by Zimmermann [19] or, more directly with the help of the generalized retarded products and their completeness equations as given in $E$.

Assume that we wish to restrict the amputated variable $P_{a}$ to the mass shell. Consider then $\hat{\tau}_{2}\left(P_{1}, \ldots, P_{a-1}, \bar{P}_{a}, p_{1}, p_{2}\right)$. This function contains the pole term

$$
\Pi\left(P_{1}, \ldots, P_{a}, p_{1}, p_{2}\right)=2 \pi \frac{\hat{\tau}_{0}\left(\ldots, P_{a-1}, \bar{P}\right) \hat{\tau}_{2}\left(\bar{P}_{a},-\bar{P}, p_{1}, p_{2}\right)}{P^{2}-M^{2}+i \varepsilon}
$$

with $P=P_{a}+p_{1}+p_{2}$. Insertion into (5.15) gives as contribution to $\hat{\tau}_{2}\left(P_{1}, \ldots, P_{a-1}, \bar{P}_{a}\right)$ :

$$
\begin{aligned}
& \Pi\left(P_{1}, \ldots, P_{a}\right)=i \pi \frac{\hat{\tau}_{0}\left(P_{1}, \ldots, P_{a-1}, \bar{P}_{a}\right)}{P_{a}^{2}-M^{2}+i \varepsilon} \\
& \quad \cdot \int d w\left\{\frac{\hat{\tau}_{2}\left(\bar{P}_{a},-\bar{P}_{a}, w,-w\right)}{w^{2}-m^{2}+i \varepsilon}-\frac{\hat{\sigma}_{2}\left(-w, w, \bar{P}_{a},-\bar{P}_{a}\right)}{w^{2}-m^{2}+i \varepsilon w_{0}}\right\} .
\end{aligned}
$$

We see that there is still a pole in the amputated function so that $P_{a}$ cannot be put onto the mass shell!

Under these circumstances our statement that (5.15) solves (5.4) has clearly to be taken with a grain of salt. It is, however, still rigorously true that

$$
\tilde{\tau}_{2}\left(\bar{P}_{1}, \bar{P}_{2}\right)-\tilde{\tau}_{2}^{*}\left(\bar{P}_{1}, \bar{P}_{2}\right)=0 \quad \text { for } \quad P_{2}^{2} \sim M^{2}
$$

for the two-point function defined by (5.15). Let

$$
\begin{aligned}
& A_{2}(P)=\left(P^{2}-M^{2}\right)^{2} \hat{\tau}_{2}(P) \\
& =\int d w\left\{\frac{\hat{\tau}_{2}(\bar{P},-\bar{P}, w,-w)}{w^{2}-m^{2}+i \varepsilon}-\frac{\hat{\sigma}_{2}(-w, w, \bar{P},-\bar{P})}{w^{2}-m^{2}-i \varepsilon w_{0}}\right\} .
\end{aligned}
$$

Because of the invariance properties of $\hat{\tau}_{2}, A_{2}$ is a function of $P^{2}$ only. Due to (6.3) it can be written

$$
A_{2}\left(P^{2}\right)=i \alpha_{2}+\left(P^{2}-M^{2}\right) \cdot \beta_{2}\left(P^{2}\right)
$$

where $\alpha_{2}=-i A_{2}\left(M^{2}\right)$ is a real constant and $\beta_{2}$ is holomorphic in a neighbourhood of $P^{2}=M^{2}$.

Consider now the function $\hat{\tau}\left(P_{1}, \ldots, P_{a}\right)$ as far as terms of second order: $\hat{\tau}_{0}+e^{2} \hat{\tau}_{2}$. It contains the following one-particle singularity in the variable $P_{a}$ :

$$
\begin{aligned}
& \frac{\hat{\tau}_{0}\left(P_{1}, \ldots, P_{a-1}, \bar{P}_{a}\right)}{P_{a}^{2}-M^{2}+i \varepsilon}\left[1-\frac{e^{2} \pi \alpha_{2}}{P_{a}^{2}-M^{2}+i \varepsilon}-e^{2} \pi \beta_{2}\right] \\
& \quad=\frac{\hat{\tau}_{0}\left(P_{1}, \ldots, P_{a-1}, P_{a}\right)}{P_{a}^{2}-M^{2}+i \varepsilon}\left(1-e^{2} \pi \beta_{2}\right)\left[1-\frac{e^{2} \pi \alpha_{2}}{P_{a}^{2}-M^{2}+i \varepsilon}\right]
\end{aligned}
$$


up to term of higer order. This form suggests that we consider $\left(P^{2}-M^{2}+i \varepsilon\right)^{-1}\left[1-e^{2} \pi \alpha_{2}\left(P^{2}-M^{2}+i \varepsilon\right)^{-1}\right]$ as the first terms of the development

$$
\begin{aligned}
& \frac{1}{P^{2}-M^{2}-\delta M^{2}}+i \varepsilon \\
& =\frac{1}{P^{2}-M^{2}+i \varepsilon}\left[1+\frac{e^{2}\left(\delta M^{2}\right)_{2}}{P^{2}-M^{2}+i \varepsilon}+\text { higher terms }\right]
\end{aligned}
$$

where $\delta M^{2}$ is a power series in $e^{2}$ with $\left(\delta M^{2}\right)_{0}=0$ and

$$
\left(\delta M^{2}\right)_{2}=-\pi \alpha_{2} .
$$

In other words, if we amputate $\tilde{\tau}\left(P_{1}, \ldots, P_{a}\right)$ with $\left(P_{a}^{2}-M^{2}-\delta M^{2}\right)$ and define the mass shell by $P_{a}^{2}=M^{2}+\delta M^{2}$, then the mass shell restriction of the amputated $\tilde{\tau}$ does exist up to second order:

$$
\begin{aligned}
& \left(P_{a}^{2}-M^{2}-\delta M^{2}\right) \tilde{\tau}=\left(P_{a}^{2}-M^{2}\right) \tilde{\tau}_{0}\left(P_{1}, \ldots, P_{a}\right) \\
& \quad-e^{2}\left(\delta M^{2}\right)_{2} \tilde{\tau}_{0}\left(P_{1}, \ldots, P_{a}\right)+e^{2}\left(P_{a}^{2}-M^{2}\right) \tilde{\tau}_{2}\left(P_{1}, \ldots, P_{a}\right)+\cdots .
\end{aligned}
$$

The first term on the right exists to second order on the new mass shell if we assume that $\tilde{\tau}_{0}\left(P_{1}, \ldots, \bar{P}_{a}\right)$ is differentiable with respect to the transversal (relative to the mass shell) component of $P_{a}$, even if some of the other $P_{j}$ are also restricted to the mass shell. This has been proved by Hepp [20] for the case of non-overlapping momenta and is a reasonable assumption even for overlapping $P$. The pole in the second term of the r.h.s. is cancelled by the pole (6.2) of the third term, whence the existence of the new mass shell restriction follows.

The mass-shifted completeness equations differ from the original ones in two respects: the integration variables $W_{j}$ have to be amputated with the new mass $M^{2}+\delta M^{2}$ and the factors $\delta_{+}\left(W_{j}\right)$ contain the new mass $M^{2}+\delta M^{2}$. This leads in (5.2) to additional terms of the form

$$
\begin{aligned}
\left(\delta M^{2}\right)_{2} \int \prod_{1}^{\alpha}\left\{d U_{j} d V_{j} \Delta_{+}\left(U_{j}-V_{j}\right)\right\} & \\
\cdot & r_{0}\left(X, X_{L}, x_{1}, x_{2}, \bar{U}_{1}, \ldots, \bar{U}_{\alpha-1}, U_{\alpha}\right) r_{0}\left(Y, X_{R}, \ldots, \bar{V}_{\alpha}\right)
\end{aligned}
$$

from the new amputation prescription and

$$
\begin{aligned}
\left(\delta M^{2}\right)_{2} \int \prod_{1}^{\alpha} d U_{j} d V_{j} \prod_{1}^{\alpha-1} \Delta_{+}\left(U_{j}-V_{j}\right) \frac{\partial}{\partial M^{2}} \Delta_{+}\left(U_{\alpha}-V_{\alpha}\right) \\
\cdot r_{0}\left(X, X_{L}, x_{1}, x_{2}, \ldots\right) r_{0}(\ldots)
\end{aligned}
$$

from the explicit $M$-dependence of the equations. Both types of term vanish because of (3.2). Hence (5.3) satisfies the mass-shifted Eq. (5.2) and from this fact we deduce that (5.15) satisfies the mass-shifted Eqs. (5.4) exactly as we did in Section $\mathrm{V}$ for the original equations. 
Equation (6.8) is then our formula for the electromagnetic mass shift of the hadron:

$\left(\delta M^{2}\right)_{2}=\left.i \pi \int d w\left\{\frac{\hat{\tau}_{2}(\bar{P},-\bar{P}, w,-w)}{w^{2}-m^{2}+i \varepsilon}-\frac{\hat{\sigma}_{2}(-w, w, \bar{P},-\bar{P})}{w^{2}-m^{2}-i \varepsilon w_{0}}\right\}\right|_{P^{2}=M^{2}}$

For $P^{2}=M^{2}$ we have

$$
\hat{\sigma}_{2}(-w, w, \bar{P},-\bar{P})=\hat{r}_{2}(-w, w, \bar{P},-\bar{P}),
$$

the difference in the prescription for taking the limit of $\mathrm{H}_{2}$ being irrelevant because $\mathrm{H}_{2}$ is analytic on the boundary in the critical variables.

Let $|P\rangle=A^{\mathrm{in}^{*}}(P) \Omega$. The LSZ reduction formulae (see $E$ ) tell us

$$
\begin{aligned}
& \langle P|\tilde{T}(p, q)| Q\rangle_{2}^{T}=(2 \pi)^{2} \delta_{+}(P) \delta_{+}(Q) \tilde{\tau}_{2}^{T}(\bar{P},-\bar{Q}, p, q), \\
& \langle P|\tilde{R}(p, q)| Q\rangle_{2}^{T}=(2 \pi)^{2} \delta_{+}(P) \delta_{+}(Q) \tilde{r}_{2}(p, q, \bar{P},-\bar{Q})
\end{aligned}
$$

whence

$$
\begin{aligned}
& \delta_{+}(P) \delta_{+}(Q) \delta^{4}(P-Q) \cdot\left(\delta M^{2}\right)_{2} \\
& \quad=\frac{i}{4 \pi} \int d w\left\langle P\left|\frac{\tilde{T}(-w, w)}{w^{2}-m^{2}+i \varepsilon}-\frac{\tilde{R}(-w, w)}{w^{2}-m^{2}-i \varepsilon w_{0}}\right| Q\right\rangle_{2}^{T} .
\end{aligned}
$$

This becomes, with the help of (2.11) and (5.8),

$$
\begin{aligned}
\delta_{+}(P) \delta_{+}(Q) \delta^{4}(P-Q) & \cdot\left(\delta M^{2}\right)_{2}=\frac{1}{2} \int d w \delta_{+}(w)\langle P|\tilde{T}(-w, w)| Q\rangle_{2}^{T} \\
+ & \frac{1}{4 \pi} \int \frac{d w}{w^{2}-m^{2}-i \varepsilon w_{0}}\left\langle P\left|B^{*}(w) B(w)\right| Q\right\rangle_{2}^{T}
\end{aligned}
$$

which is our final result.

\section{Discussion}

We have developed a form of perturbation theory for an interaction of electromagnetic type which takes the strong interactions rigorously into account. As a result we have obtained the expression (6.14) for the second-order electromagnetic mass shift of hadrons. This expression must be compared with the form (1.1), which reads for our model and in our notation,

$\delta_{+}(P) \delta_{+}(Q) \delta^{4}(P-Q) \cdot\left(\delta M^{2}\right)_{2}^{\prime}=\frac{i}{4 \pi} \int \frac{d w}{w^{2}-m^{2}+i \varepsilon}\langle P|\tilde{T}(-w, w)| Q\rangle_{2}^{T}$,

and which we would have obtained if (5.5) had been the correct form of $\tilde{\tau}_{2}^{T}$. 
An obvious advantage of (6.14) over (7.1) is that the ambiguity inherent in the definition of the $T$-product is completely irrelevant. The $T$-product occurs only in the first term, where both the hadron momentum $P$ and the photon momentum $w$ lie on their respective mass shell. There $\langle P|\tilde{T}(-w, w)| Q\rangle$ is unambiguously defined as the physical forward Compton scattering amplitude. (Remember that the photon variables $w$ and $-w$ are to be amputated.) In the second, off-mass-shell, term only the absorptive part of this amplitude enters and this is defined unambiguously in terms of the fields.

The two expressions differ by the term

$$
\Delta=\frac{i}{4 \pi} \int \frac{d w}{w^{2}-m^{2}-i \varepsilon w_{0}}\langle P|\tilde{R}(-w, w)| Q\rangle_{2}^{T} .
$$

The integrand is analytic in the lower half $w_{0}$-plane. Hence $\Delta$ vanishes if the $w_{0}$ integration contour can be closed by an infinite semicircle in the lower half plane. The remarks made after Eq. (5.11) apply here: it may very well happen that $\left(\delta M^{2}\right)_{2}$ is finite and yet $\Delta \neq 0$. In this case $\left(\delta M^{2}\right)_{2}$ is the correct value of the mass shift, $\left(\delta M^{2}\right)_{2}^{\prime}$ is wrong. We remind the reader that this situation may occur if the absorptive part shows an oscillatory behaviour for large $w_{0}$.

If $\Delta=0$, then $\left(\delta M^{2}\right)_{2}=\left(\delta M^{2}\right)_{2}^{\prime}$. It is, however, important to note how the integral $\Delta$ is defined. The following situation is of practical importance: assume that the integrand $I(w)$ of (7.2), considered as a function of $w_{0}$, is bounded for large $w_{0}$ by $c(\boldsymbol{w})\left|w_{0}\right|^{-1-\varepsilon},(\varepsilon>0)$, where $c(\boldsymbol{w})$ is not strongly decreasing for large $|\boldsymbol{w}|$. Then

$$
\lim _{R \rightarrow \infty} \int d^{3} w \int_{-R}^{R} d w_{0} I(w)=0
$$

However,

$$
\lim _{R \rightarrow \infty} \int_{-R}^{R} d w_{0} \int d^{3} w I(w)
$$

need not even be defined, much less vanish. In this case the equality $\left(\delta M^{2}\right)_{2}=\left(\delta M^{2}\right)_{2}^{\prime}$ holds only if the right-hand side is defined as $\int d^{3} w \int d w_{0} \ldots$, and the integrations must not be interchanged without full justification.

The reader might be inclined to find our derivation of (6.14) not too convincing. In particular, he might distrust the admittedly rather vague remarks on the existence of solutions distinguished by their optimal behaviour at infinity. We should therefore like to point out that our rejection of (7.1) does not rest on these arguments but on the simple 
fact that it violates unitarity (if $\Delta \neq 0$ ). It is therefore inadmissible also in a canonical framework.

Another comment that ought to be made is this: it is well known that $\delta M^{2}$ is not measurable, since the electromagnetic interactions cannot be switched off experimentally. One can measure only mass differences between hadrons whose masses are expected to coincide in the limit $e \rightarrow 0$. Let us then consider a theory with two hadrons $A_{1}$ and $A_{2}$, both with the same zero-order mass $M$. It might happen that the individual mass shifts turn out to be infinite, but that their difference is finite. This situation seems to be quite satisfactory since the mass difference is anyhow the only observable quantity in the problem. However, things are not really that good because our formalism would then start from a 0 th-order theory describing strongly interacting particles of infinite mass, a fact which would certainly detract somewhat from the credibility of our procedure.

Finally we wish to make two remarks about our perturbation theory in itself, without regard to the calculation of mass shifts.

a) We might decide to apply perturbation theory also to the strong interactions and study things in every order of this. The Feynman rules then clearly tell us that (5.5) is the correct form of $\tilde{\tau}_{2}^{T}\left(P_{1}, \ldots, P_{a}\right)$. Do our results, then, imply that the Feynman rules are wrong? No, because the $\tau$-functions of perturbation theory never show an oscillatory behaviour at infinity. Therefore, either both (5.5) and (5.15) diverge, or both are convergent and equal, so that no contradiction is present.

b) The whole procedure becomes questionable if the hadron turns out to be unstable under electromagnetic interactions, for then the structure of the Hilbert space $\mathfrak{H}$ gets changed by the switching-on of the electromagnetic interactions, i.e., Eq. (2.1) is no longer correct. Such an instability occurs in second order if the vertex function $\tilde{\tau}_{2}\left(\bar{P}, p_{1}, p_{2}\right)$ does not vanish for $P^{2}=M^{2}, p_{1}^{2}=p_{2}^{2}=m^{2}$.

Let $|P\rangle^{\mathrm{nn} \text {, out }}$ be a state with one incoming (outgoing) hadron of momentum $P,\left|p_{1}, p_{2}\right\rangle^{\text {in, out }}$ analogously a two-photon state. Then we have in our case

$$
{ }^{\text {out }}\left\langle p_{1}, p_{2} \mid P\right\rangle_{2}^{\text {in }} \neq 0 \text {. }
$$

$|P\rangle^{\text {in }}$ is no longer a stationary state, as stable one-particle states have to be. However, if we calculate in $\left\langle p_{1}, p_{2} \mid P\right\rangle_{2}^{\text {in }}$ by summing over a complete set of intermediary out-states, we find that this expression still vanishes, so that (2.1) remains true in second order. This is related to the fact that $\left(\delta M^{2}\right)_{2}$ is a real number, i.e., the shifted hadron pole in the propagator still lies on the real axis and the cut starting at $(2 m)^{2}$ is not yet present in second order. 
Acknowledgments. Part of this work was carried out during a stay at the Institute for Theoretical Physics of the Free University of Berlin. The author wishes to thank Professors F. Penzlin and W. Theis (Berlin) and Professors Abdus Salam and P. Budini (Trieste) and the International Atomic Energy Agency for the hospitality accorded to him at their respective institutions. He is indebted to Professor Theis for rousing his interest in the problem of mass shifts and for several helpful discussions.

\section{References}

1. Bjorken, J. D.: Phys. Rev. 148, 1467 (1966).

2. Brandt, R., and J. Sucher: Phys. Rev. 177, 2218 (1969).

3. Cini, M., E. Ferrari, and R. Gatto: Phys. Rev. Letters 2, 7 (1959).

4. Barger, V., and E. Kazes: Nuovo Cimento 28, 385, 394 (1963).

5. Riazuddin and Fayyazuddin: Phys. Rev. 158, 1447 (1967).

6. - Phys. Rev. 114, 1184 (1959).

7. Theis, W. R.: Nucl. Phys. 21, 170 (1960).

8. Nishijima, K.: Phys. Rev. 119, 485 (1960).

9. Steinmann, O.: Ann. Phys. (N. Y.) 29, 76 (1964); 36, 267 (1966).

10. Streater, R. F., and A. S. Wightman: PCT, spin and statistics and all that. New York: W. A. Benjamin, Inc. 1964.

11. Jost, R.: The general theory of quantized fields. Am. Math. Soc., Providence 1965.

12. Steinmann, O.: Commun. Math. Phys. 10, 245 (1968).

13. Glaser, V., H. Lehmann, and W. Zimmermann: Nuovo Cimento 6, 1122 (1957).

14. Ruelle, D.: Nuovo Cimento 19, 356 (1961).

15. Araki, H.: J. Math. Phys. 2, 163 (1961).

16. Epstein, H.: In: Axiomatic field theory, ed. by M. Chrétien and S. Deser. New York: Gordon \& Breach 1966.

17. Borchers, H. J.: Nuovo Cimento 15, 784 (1960).

18. Zimmermann, W.: Commun. Math. Phys. 6, 161 (1967).

19. - Nuovo Cimento 13, 503 (1959); 16, 690 (1960).

20. Hepp, K.: Commun. Math. Phys. 1, 95 (1965).

O. Steinmann

International Centre for Theoretical Physics

Miramare, P. O. Box 586

I 34100 Trieste 